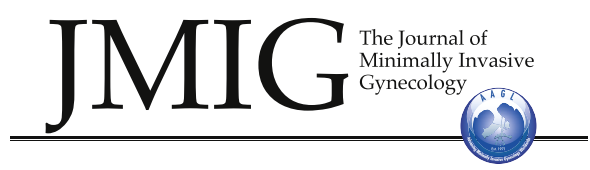

Instruments and Techniques

\title{
A Simple Laparoscopic Procedure to Restore a Normal Vaginal Length After Colpohysterectomy With Large Upper Colpectomy for Cervical and/or Vaginal Neoplasia
}

\author{
Eric Leblanc, MD*, Lucie Bresson, MD, Benjamin Merlot, MD, Marco Puga, MD, \\ Frederic Kridelka, MD, PhD, Audrey Tsunoda, MD, and Fabrice Narducci, MD \\ From the Department of Gynecologic Oncology (Drs. Leblanc, Bresson, Merlot, Puga, and Narducci), Centre Oscar Lambret, Lille, France, Department of \\ Gynecologic Oncology (Dr. Kridelka), CHU ND des Bruyères, Chênée-Liège, Belgium, and Department of Gynecologic Oncology (Dr. Tsunoda), Barretos \\ Cancer Center, Barretos, Brazil.
}

\begin{abstract}
Colpohysterectomy is sometimes associated with a large upper colpectomy resulting in a shortened vagina, potentially impacting sexual function. We report on a preliminary experience of a laparoscopic colpoplasty to restore a normal vaginal length. Patients with shortened vaginas after a laparoscopic colpohysterectomy were considered for a laparoscopic modified Davydov's procedure to create a new vaginal vault using the peritoneum of the rectum and bladder. From 2010 to 2014,8 patients were offered this procedure, after informed preoperative consent. Indications were 2 extensive recurrent vaginal intraepithelial neoplasias grade 3 and 6 radical hysterectomies for cervical cancer. Mean vaginal length before surgery was $3.8 \mathrm{~cm}$ (standard deviation, 1.6). Median operative time was 50 minutes (range, 45-90). Blood loss was minimal (50-100 mL). No perioperative complications occurred. Median vaginal length at discharge was $11.3 \mathrm{~cm}$ (range, 9-13). Sexual intercourse could be resumed around 10 weeks after surgery. At a median follow-up of 33.8 months (range, 2.4-51.3), 6 patients remained sexually active but 2 had stopped. Although this experience is small, this laparoscopic modified Davydov's procedure seems to be an effective procedure, adaptable to each patient's anatomy. If the initial postoperative regular self-dilatation is carefully observed, vaginal patency is durably restored and enables normal sexual function. Journal of Minimally Invasive Gynecology (2015) $\square, \boldsymbol{\square}-\mathbf{\square}$ (C) 2015 AAGL. All rights reserved.
\end{abstract}

Keywords: $\quad$ Colpohysterectomy; Colpoplasty; Davydov; Laparoscopy; Vaginoplasty DISCUSS You can discuss this article with its authors and with other AAGL members at http://
www.AAGL.org/jmig-22-6-JMIG-D-15-00319.

\section{Introduction}

Radical colpohysterectomy is the cornerstone of surgical management of early cervical carcinoma IB or IIA. Radical colpectomy can be an option in the management of upper vaginal malignancies or recurrence. Both procedures often result in a more or less generous resection of the upper part of the vagina.

\footnotetext{
None of the authors has a financial interest in any of the products, devices, or drugs mentioned in this article.

Corresponding author: Eric Leblanc, MD, Department of Gynecologic Oncology, Centre Oscar Lambret, Lille, France.

E-mail: e-leblanc@o-lambret.fr
}

Submitted May 26, 2015. Accepted for publication August 12, 2015.

Available at www.sciencedirect.com and www.jmig.org
According to reports from patients and their sexual partners, quality of life may be seriously impaired because of a shortened vagina. Current methods to solve this problem are not always satisfactory and vary from the simple use of dilators to complex surgical techniques such as intestinal colpoplasties or vaginoplasties, using different myocutaneous flaps.

In this article we present a new and simple method that can be performed by either a laparoscopic or an open approach, at completion of a radical surgery, or secondarily. The technical principle is based on Davydov's procedure, initially designed to treat vaginal aplasia. Because it mainly consists of a local peritoneoplasty, it has little risk of bowel toxicity or aesthetic sequelae. We describe our initial experience using a laparoscopic approach. 


\section{Methods}

After completion of the colpectomy, vaginal length was measured from the vaginal cut edge to the superficial aspect of labia majora (because hymeneal remnants were not always visible). If the length was less than or equal to $5 \mathrm{~cm}$, the modified Davydov's procedure was considered. Informed consent was obtained from all enrolled patients, and the study was approved by our Institutional Review Board.

\section{Technique}

The peritoneum of the bladder base was sutured onto the anterior edge of the vagina by interrupted stitches (Vicryl nr0 or 1; Ethicon, Somerville, NJ) or a continuous suture (Vicryl or V-lock nr2.0 suture; Covidien-Medtronic, Minneapolis, MN). Posteriorly, the anterior peritoneum of the pouch of Douglas was sutured onto the posterior edge of the vagina using the same material. When the vagina was very short, we mobilized the bladder to enable the creation of a neovagina by detaching it from the anterior abdominal wall by division of the obliterated urachus (Fig. 1A-C).

A vaginal probe was then introduced through the remaining vagina into the pelvic cavity. We initially used a standard glass dilator offered by radiation therapists to patients for post-treatment care. Currently, we use silicon vaginal dilators (Vagiwell MEDIntim; Mörfelden, Waldorf, Germany), initially designed for vaginal aplasia correction, because they are more comfortable for patients. A dilator was selected among 5 sizes (ranging from 122 to $177 \mathrm{~mm}$ long and 14 to $30 \mathrm{~mm}$ diameter) to fit the patient's morphology. This dilator was introduced as high as possible, leaving only the basis of the device outside the labia, for a stable fixation onto the perineal skin.

Laparoscopically, the bladder peritoneum and rectal serosa were then sutured together over the tip of the probe to create the new vault. Laterally, the edges of both the lateral rectal and vesical peritoneum were fixed together around the probe to create a peritoneal cylinder that completely wraps around the probe. One can use interrupted or continuous resorbable sutures (Fig. 2A-C). This step requires careful attention, especially in patients who have had previous surgery, because it is important to avoid including the ureters into the lateral sutures.

The operation was completed when the vaginal probe was no longer visible internally (Fig. 3). Drainage was not necessary. Initially, glass probes were fixed to the perineum with dressing us- ing Elastoplast ${ }^{\circledR}$ (BSN medical, Charlotte, NC), but patients were obliged to stay on bedrest for 9 days. The design of the current silicon probes enables a fixation with direct sutures to the perineal skin so the patient can freely mobilize. Mobilization was encouraged by the second or third postoperative day, allowing the seating position with caution.

The probe was left in place for 9 to 10 days, along with the bladder catheter. Daily thromboprophylaxis was prescribed during the postoperative period and continued for 3 postoperative weeks. Some local care was necessary to keep the surgical area clean. On the 10th day the probe was detached and removed, along with the bladder catheter. A thorough vaginal washing was then performed. The patient was then taught how to perform selfdilatation of her neovagina using the same probe; a 30-minute session at least 2 to 3 times a day for 2 months was advised.

After 8 weeks the reconstructed vaginal vault was clinically evaluated during a pelvic examination with speculum. A Pap smear was performed to check the presence of normal squamous cells; if healing seemed to be complete, resumption of vaginal intercourse was encouraged. If healing seemed to be incomplete, resumption of vaginal intercourse was delayed until the next follow-up visit, scheduled every 3 weeks, to confirm satisfactory healing. Then, routine follow-up oncology visits were performed for 5 years from initial treatment.

\section{Results}

Between January 2010 and February 2014, 8 patients were offered this procedure. Two had extensive and recurrent vaginal intraepithelial neoplasia (VAIN) grade 3 lesions, whereas the other 6 patients suffered from cervical carcinoma. Median age was 41 years (range, 23-65), median body mass index was $19.2 \mathrm{~kg} / \mathrm{m}^{2}$ (range, 16.2-36). Patient characteristics are summarized in Table 1 . Seven patients underwent a laparoscopic approach, and 1 patient was operated on using the Da Vinci (Intuitive Surgical Inc, Sunnyvale, CA.) robotic platform, following the same technical principles.

Specific duration of the procedure averaged 43 minutes (range, 30-70), according to the length of the new vaginal vault. No significant blood loss was observed during this step. Mean hospital stay was 10 days (range, 9-15).

\section{Fig. 1}

Different steps in the laparoscopic creation of a modified Davydov's peritoneal neovagina. (A) Anterior vesicovaginal suture using the peritoneum of the bladder base. (B) Posterior rectovaginal suture using the peritoneum of the Douglas pouch. (C1) Urachus division to enable. (C2) Bladder corpus mobilization.
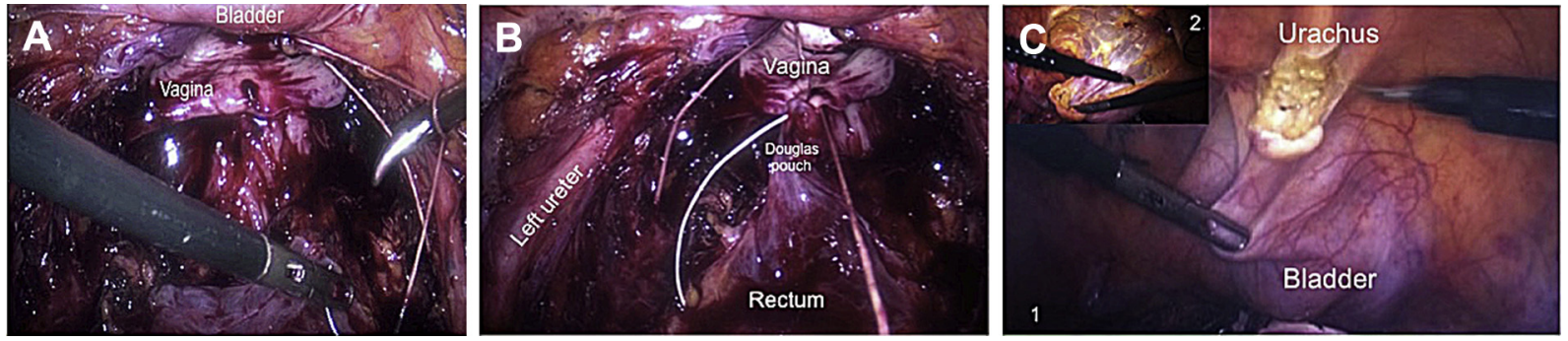


\section{Fig. 2}

Creation of the peritoneal vault. (A) Suture of both rectal and vesical peritoneum over a vaginal probe. Left (B) and right (C) lateral peritoneal are sutured over the probe.
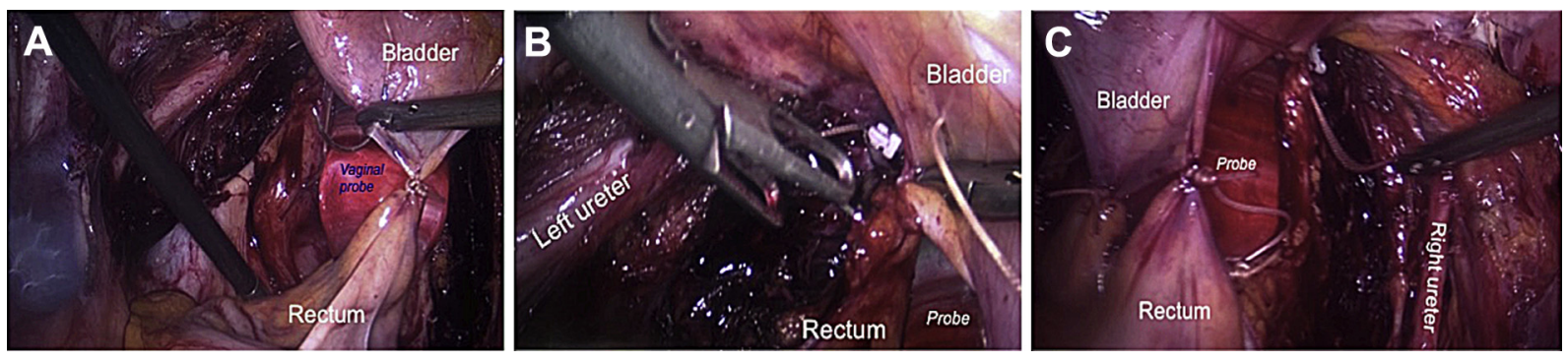

One patient developed, on the seventh postoperative day, an intestinal volvulus that was treated laparoscopically. No abnormality was observed at the operative site. Dilators were all removed on the 9th or 10th postoperative day. Length of the vagina (measured with a ruler from the top of the vault to labia majora) averaged $11.4 \mathrm{~cm}$ (range, 10-15), with variations related to probe size.

Postoperative outcomes are summarized in Table 2. Postoperatively, the vaginal length was slightly reduced from $11 \mathrm{~cm}$ (range, 4-13) at 2 months to $10.3 \mathrm{~cm}$ (range, 5-10) at 6 months. No patient to date developed bowel or urinary complications.

With a median follow-up of 35.8 months (range, 5.454.3), we observed 1 recurrence of the initial disease, and 1 patient developed another primary tumor. One of 2 patients with VAIN3 persisted with oncogenic human papillomavirus and developed new lesions of VAIN3 at the 12-month follow-up. She eventually received contact brachytherapy, and at 51.3 months and although she remained free of vaginal disease, she decided to stop vaginal intercourse.

\section{Fig. 3}

Davydov's inspired neovaginal vault: final aspect. (A) Final external view with the glass probe $(p)$ installed into the neovagina. (B) Final laparoscopic view of the peritoneum of the mobilized bladder and the anterior rectum, completely wrapping the glass probe between both ureters $(U)$.

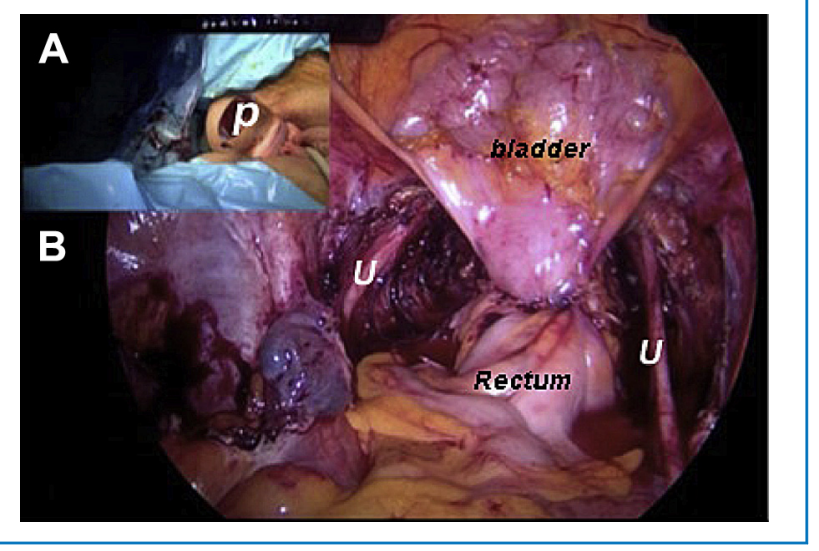

The other patient was primarily managed by a combination of brachytherapy and colpohysterectomy and modified Davydov's procedure for a cervical cancer IB1. Unfortunately, she developed a urothelial bladder carcinoma with pulmonary metastases at 15 months and is currently receiving chemotherapy. The other 6 patients are free of disease so far.

Because of the very preliminary nature of this series, no standardized questionnaire of sexual satisfaction was completed by the patients. The level of satisfaction through history-taking, along with neovagina measurement during the consultation, was reported. In the future a validated patient questionnaire will be offered to such patients. All except 1 patient resumed sexual activity with a median delay of 10 weeks (range, 8-15). Vaginal sensation was reported as "minimal," but penetration was described as satisfactory and painless in 6 of 7 patients, along with normal vulvar sensitivity. The use of vaginal lubricants was helpful for 3 patients.

The patient with recurrent VAIN3 had a shortened but patent vagina $(4 \mathrm{~cm})$. Intercourse was initially possible although slightly painful but was stopped due to both the treatment of this recurrence and the concurrent diagnosis of a breast cancer. The patient with urothelial carcinoma abruptly stopped using her dilator because she had no sexual activity. Her vaginoplasty completely shrank, with a residual vaginal length of $3 \mathrm{~cm}$. The other 6 patients are free of disease and maintained a satisfactory sexual life with regular vaginal intercourse. Only the most recent case is still using dilators occasionally.

\section{Discussion}

Although still poorly addressed in the literature, vaginal shortening seems a significant complication of the surgical treatment of cervix and/or vaginal neoplasia and is a source of dyspareunia or dehiscence [1,2]. However, the specific sexual impact of this situation is variable according to the patient and her partner [3]. It seems especially problematic after radiation therapy and is inconsistently prevented by the use of dilators [4].

Multiple methods of vaginal replacement, using digestive segments, omental flaps, or various myocutaneous or 


\begin{tabular}{|c|c|c|c|c|c|c|}
\hline 2 & 62 & 16.2 & Squamous cervical cancer FIGO IB1: RH post-C-V BT & 5 & 11 & 0 \\
\hline 3 & 65 & 18.4 & $\begin{array}{l}\text { VAIN3: extended vaginal lesions; recurrence (iterative phototherapies } \\
\text { and local excisions): } \mathrm{SH} \text { with large superficial upper colpectomy }\end{array}$ & 2.5 & 11 & $\begin{array}{l}\text { Small bowel torsion } \\
\text { laparoscopic } \\
\text { detorsion }\end{array}$ \\
\hline 4 & 39 & 19.6 & $\begin{array}{l}\text { Squamous cervical cancer FIGO IB1 with positive pelvic nodes: RH } \\
\text { after definitive pelvic CRT }\end{array}$ & 4 & 12 & 0 \\
\hline 6 & 27 & 17.9 & Squamous cervical cancer: FIGO IB1: RH post-C-V BT & 3 & 12 & 0 \\
\hline 7 & 23 & 31.2 & $\begin{array}{l}\text { Clear cell cervical cancer at } 9 \text { years old: post-C-V BT upper vagina } \\
\text { stenosis: SH along with large upper vagina resection }\end{array}$ & 4 & 15 & 0 \\
\hline 8 & 40 & 36 & $\begin{array}{l}\text { Squamous cervical cancer FIGO IIIA: RH post-CRT with shortened } \\
\text { vagina }\end{array}$ & 3 & 15 & 0 \\
\hline
\end{tabular}

fasciocutaneous flaps, have been reported to solve this problem. These techniques are generally used after exenterative procedures to both create a vagina and fill up the empty pelvis. Recently, a laparoscopic treatment of a vaginal carcinoma with vaginoplasty using a sigmoid segment was reported in 5 patients with good results [5]. However, this complex approach requires a large bowel resection with potential serious complications. In addition, colonic vaginoplasty is often a source of permanent and uncomfortable discharge [6].

Our aim was to find a simple method of vaginal vault repair that can be performed through laparoscopy with bladder and rectum in place. We investigated surgical methods for corrections of vaginal aplasia (Mayer-Rokitansky-Kuster syndrome). Although Vecchietti's mechanical method [7] was obviously not adapted to our patients' situation, McIndoe's technique, consisting of the placement, between the rectum and vagina, of a mold covered by a splitthickness skin graft, which becomes the future neovagina, was considered [8]. The disadvantages of this procedure are the need to sample skin, creating a new visible scar(s), and the necessity of a long and strict bedrest to avoid graft mobilization and necrosis. The durable and regular use of dilators is recommended long afterward to maintain the

\section{Table 2}

Late patients' outcomes

Vaginal size

at 2 months

(cm)

Vaginal intercourse

Vaginal size

Temporary (4 mo until BC)

12 months (cm)

Pap smear

9

No

5

Yes satisfactory

Yes satisfactory

Stenosis at $12 \mathrm{mo}$

10

Yes satisfactory

10

9

Yes satisfactory

11

Yes satisfactory

10

Yes satisfactory

(2-6 months)

Cancer recurrence

Status

Normal/VAIN3

\section{Breast cancer}

NED

Normal/normal Urothelial carcinoma + pulmonary metastasis NED

Normal/normal 0 NED

Normal/normal 0 NED

Normal/normal $0 \quad$ NED

Normal/normal 0

Normal/normal 0

Normal/normal 0

NED 
neovaginal patency. Some recent refinements of this old technique, using glue associated with vacuum therapy, seem to have improved functional results [9].

The Wheeless's procedure [10] consists of the attachment of a harvested omental J-flap onto the remaining vagina. A dilator maintains the volume of this omental cylinder. A skin graft is placed at the time of the procedure or secondarily to cover the inner part. The issue of skin scarring due to grafting is similar to the McIndoe procedure. In addition, the omentum is not always suitable for this technique because of a variable volume.

Davydov's technique was then considered. In the original report the upper part of a neovagina was created by using the peritoneum of the Douglas pouch, whereas a bilateral nymphoplasty, from both labia minora, was performed to create the inferior cutaneous part of the neovagina [11]. If only the upper part of the vagina needs to be restored (without the need for a nymphoplasty), the Davydov procedure is ideal [12]. This procedure can be easily performed by laparoscopy [13], with no need of skin grafting, flap, or any foreign material as a mesh. The presence of normal vaginal tissue in the inferior part is important to enable re-epithelialization of the peritoneal surfaces of the upper colpoplasty by squamous cells. In addition, interesting long-term results on sexual function after Davydov's technique in vaginal aplasia were previously described [14].

As for any vaginoplasty using peritoneum or skin graft, the critical point is the observance of regular postoperative self-dilatations, until quite complete vault re-epithelialization by normal squamous vaginal cells is obtained, thus preventing secondary stenosis. It takes around 2 months, and thereafter resumption of vaginal intercourse is encouraged, because it is the best way to maintain vaginal patency. The use of a local estrogen therapy, although not used in the present series, may accelerate healing and improve comfort. An alternative is the use of biologic grafts to create such a vault. However, this material is still very expensive and was not available in our institution. A recent article, in spite of few adverse effects, reported a high satisfaction rate in a population of patients with Mayer-Rokitansky-Kuster syndrome [15].

The indications of this laparoscopic modified Davydov's procedure are not frequent (explaining the small number of our series, spanning over 4 years) because of they are limited to large upper colpectomies in sexually active patients. The main indication is radical colpohysterectomy for an invasive cervical carcinoma, especially for FIGO stage IIA1 disease, or in case of upper third vaginal invasive carcinomas. Indeed, in these situations a large upper vaginectomy is oncologically necessary.

More specific to France is the preoperative application of $60 \mathrm{~Gy}$ of pulse dose rate cervicovaginal brachytherapy for FIGO IB1-IIA1 cervical tumors of more than $2 \mathrm{~cm}$. This policy leads to tumor shrinkage (and often to tumor sterilization) to reduce the need for extensive paracervicectomy and its specific adverse effects. However, it entails significant irra- diation of the upper vagina, and a resection of 2 to $3 \mathrm{~cm}$ of upper vagina is often necessary to prevent secondary dehiscence or healing problems. This explains the indication of this procedure for 4 patients in this series with a preoperative short vagina. Locally advanced cervix carcinomas are usually managed by definitive chemoradiation therapy or neoadjuvant chemotherapy and surgery. Radical colpohysterectomy may be indicated in case of incomplete response and is usually associated with a large colpectomy.

In all these situations, when at completion of the radical procedure the vagina is less than $5 \mathrm{~cm}$ long (from the upper section down to the surface of labia majora), in a sexually active patient, modified Davydov's procedure, as described above, can be performed in continuity with the laparoscopic radical surgery. It does not confer a significantly longer operative time or significant additional blood loss. The risk of urinary tract injury (bladder/ureters) is minimal if the peritoneal mobilization is gently and precisely performed. Of course, all patients should be preoperatively informed of this possibility.

As reported in this experience, it can be offered secondarily as well. Indeed, some patients complain of impaired sexual function because of a short vagina or even postcoital vaginal dehiscence. In these situations the complete reopening of the vaginal vault is necessary to perform this procedure.

Besides indications for invasive tumors, this procedure can be offered as well to patients with extensive high-grade VAIN, especially when all noninvasive methods have failed to provide a cure. This procedure can be performed after a large, colposcopy-guided, upper colpectomy (along with hysterectomy, if the uterus is still present). In contrast, this technique cannot be applied after a total vulvocolpectomy, because it is impossible to bring the rectal and bladder peritoneum down to the perineum. In cases of VAIN3, if fertility is to be preserved, other methods should be considered because, at present, the uterus must be removed. Similarly, this procedure is not applicable, as described, after a pelvic exenteration, because the presence of both vesical and rectal peritoneum is mandatory. However, if technically feasible, an omental flap may partially contribute to create the anterior or posterior part of Davydov's procedure. Otherwise, all alternative methods of vaginal reconstruction addressed above should be discussed on an individual basis. Although proper sexual function is not only based on the size of reproductive organs, it is obvious that a shortened vagina may be an organic obstacle for satisfactory intercourse.

The present experience of this procedure is still limited. However, the laparoscopic modified Davydov's technique presented here seems to provide a simple and promising method to restore a normal vaginal length after any indication of large upper colpectomy.

\section{Acknowledgments}

We thank Stephanie Kuku, MD (Imperial College Healthcare NHS Trust, London, UK), for the language editing of this article. 


\section{References}

1. Ye S, Yang J, Cao D. A systematic review of quality of life and sexual function of patients with cervical cancer after treatment. Int J Gynecol Cancer. 2014;24:1146-1157.

2. Aerts L, Enzlin P, Verhaeghe J. Long-term sexual functioning in women after surgical treatment of cervical cancer stages IA to IB: a prospective controlled study. Int J Gynecol Cancer. 2014;24:1527-1534.

3. Occhino JA, Trabuco EC, Heisler CA. Changes in vaginal anatomy and sexual function after vaginal surgery. Int Urogynecol J. 2011;22:799-804.

4. Miles T, Johnson N. Vaginal dilator therapy for women receiving pelvic radiotherapy. Cochrane Database Syst Rev. 2010;CD007291.

5. Yin D, Wang N, Zhang S. Radical hysterectomy and vaginectomy with sigmoid vaginoplasty for stage I vaginal carcinoma. Int J Gynaecol Obstet. 2013;122:132-135.

6. Hage JJ, Karim RB, Asscheman H. Unfavorable long-term results of rectosigmoid neocolpopoiesis. Plast Reconstr Surg. 1995;95: 842-848. discussion 849-850.

7. McIndoe A. The treatment of congenital absence and obliterative conditions of the vagina. Br J Plast Surg. 1950;2:254-267.

8. Veronikis DK, McClure GB, Nichols DH. The Vecchietti operation for constructing a neovagina: indications, instrumentation, and techniques. Obstet Gynecol. 1997;90:301-304.
9. Fussey JM, Luesley DM, Sterne GD. Vaginoplasty: a modern approach. A report of 2 cases. J Reprod Med. 2013;58:441-444.

10. Wheeless CR Jr. Neovagina constructed from an omental J flap and a split thickness skin graft. Gynecol Oncol. 1989;35: 224-226.

11. Davydov SN, Zhvitiashvili OD. Formation of vagina (colpopoiesis) from peritoneum of Douglas pouch. Acta Chir Plast. 1974;16: $35-41$.

12. Leblanc E. How I do ... a vaginal vault reconstruction after radical surgery. Gynecol Obstet Fertil. 2010;38:147-148 [In French].

13. Adamyan L. Laparoscopic management of vaginal aplasia with or without functional non communicating rudimentary uterus. In: Arregui ME, Fitzgibbons RJ, Katkhouda RJ, editors. Principles of Laparoscopic Surgery. Basic and Advanced Techniques. SpringerVerlag; 1995.

14. Giannesi A, Marchiole P, Benchaib M. Sexuality after laparoscopic Davydov in patients affected by congenital complete vaginal agenesis associated with uterine agenesis or hypoplasia. Hum Reprod. 2005;20: 2954-2957.

15. Zhu L, Zhou H, Sun Z. Anatomic and sexual outcomes after vaginoplasty using tissue-engineered biomaterial graft in patients with Mayer-Rokitansky-Kuster-Hauser syndrome: a new minimally invasive and effective surgery. J Sex Med. 2013;10:1652-1658. 